\title{
AGENESIA UNILATERAL DE CORNO UTERINO EM CADELA: CONSIDERAÇÕES REPRODUTIVAS, CIRÚRGICAS E HISTOPATOLOLÓGICAS
}

(Unilateral uterine agenesis in a bitch: reproductive consideration, surgical sterilization and histopathological description)

Fabíola Dalmolin¹, Saulo Tadeu Lemos Pinto Filho², Rafael Colomé Beck ${ }^{3}$, Hellen Fialho Hartmann², Rafael Almeida Fighera², Maurício Veloso Brun².

1Programa de Pós-Graduação em Saúde, Bem-estar e Produção Animal Sustentável, Universidade Federal da Fronteira Sul, RS, Brasil, ${ }^{2}$ Programa de Pós-graduação em Medicina Veterinária da Universidade Federal de Santa Maria, RS, Brasil, ${ }^{3}$ Universidade Federal do Rio Grande do Sul, RS, Brasil

1Autor para correspondência: fabiola.dalmolin@uffs.edu.br

RESUMO - A agenesia unilateral de corno uterino é alteração incomum em cadelas e, embora algumas vezes relatada, as informações ainda são escassas. Foi atendida uma fêmea canina primípara, sem raça definida, encaminhada para ováriohisterectomia eletiva. Durante a realização do procedimento verificou-se a ausência do corno uterino esquerdo, e por meio de avaliação anatomo-histopatológica confirmou-se agenesia de corno uterino unilateral. O objetivo deste trabalho é salientar observações acerca dos aspectos reprodutivos de uma cadela portadora de agenesia unilateral de corno uterino, postuladas por autores, assim como a conduta cirúrgica e a avaliação histopatológica aplicados no caso em questão. Palavras-chave: anomalias uterinas; gestação; ovário-histerectomia; reprodução em caninos.

ABSTRACT - Uterine horn agenesis is an uncommon alteration in bitches and although it is sometimes related, information is still scarce. It was received a mongrel canine, primiparous female referred for elective ovariohysterectomy. During the procedure it was verified the absence of the left uterine horn. Histopathological exam confirmed unilateral horn uterine agenesis. The main objective of this report is to relate some reproductive aspects, postulated by other authors, as well as surgical procedure and histopathological evaluation of a uterine horn agenesis in a dog.

Keywords - Canine reproduction; ovariohysterectomy; pregnancy; uterine anomalies. 


\section{INTRODUÇÃO}

Anormalidades congênitas do útero são raras em cães e gatos (Stone, 2003) e incluem hipoplasia, agenesia, atresia, aplasia segmentar, corpo uterino septado, cervix dupla e fusão cornual (Wykes e Olson, 1996; Lyle, 2003; Stone, 2003), sendo a prevalência da anormalidade de 0,02 - 0,05\% na população canina (Mclntyre et al., 2010).

Dentre as anomalias congênitas, a agenesia de corno uterino ou útero unicórnio é a mais frequente (Wykes e Olson, 1996) e pode ser diagnosticada durante a ováriohisterectomia ou constitui achado de necropsia, com índices de 1:5.000 até 1:10.000 nesta última. Na maioria das vezes, o ovário ipsilateral pode ser pequeno ou inexistente, e se presente, poderá localizar-se mais cranialmente que o normal, próximo ao diafragma (Stone, 2003).

Agenesia é a falha no desenvolvimento da estrutura de um órgão ou sistema devido ao não aparecimento da célula originária na formação embrionária (Wykes e Olson, 1996). Os ductos paramesonéfricos vão originar a maior parte do trato genital feminino. A agenesia de corno uterino ocorre quando há falha no desenvolvimento de um ducto paramesonéfrico, resultando em útero unicornual com a tuba uterina correspondente (Aguirra et al., 2014). Pode haver pequena banda fibromuscular no local do corno ausente, a qual pode estar acompanhada de agenesia renal unilateral (Stone et al., 1998).

congênitas $\begin{gathered}\text { Todas as anormalidades } \\ \text { estão presentes }\end{gathered}$ nascimento, embora a maioria não seja reconhecida até que 0 animal seja utilizado para reprodução ou em caso de esterilização cirúrgica (Lyle, 2003). A severidade dos defeitos pode variar desde a inexistência de sinais clínicos até alterações na capacidade reprodutiva (Colaço et al., 2012).

Durante 0 procedimento cirúrgico de ovário-histerectomia, o útero unicórnio poderá ser fator complicante, já que a ausência de um corno uterino altera a anatomia cirúrgica (Pinto Filho et al.; 2001; Stone et al., 2003; Vince et al., 2011; Pagliuca et al., 2012).

O objetivo deste relato é trazer contribuições acerca do histórico reprodutivo, o procedimento cirúrgico de ovário-histerectomia e as características histopatológicas do útero de uma fêmea canina com agenesia unilateral de corno uterino, a fim de concorrer com informações referentes às anomalias uterinas e aspectos reprodutivos em cães.

\section{RELATO DE CASO}

Foi encaminhada uma fêmea canina sem raça definida, de quatro anos de idade e 10,3 kg para ováriohisterectomia eletiva. A paciente tinha histórico de ter recebido progestágeno com objetivo de evitar gestação antes de apresentar sinais de estro. Embora os tutores não tenham verificado a presença de sinais comportamentais da fêmea, a presença de cão macho no local ou a cópula, a primípara entrou em trabalho de parto e concebeu uma ninhada sem auxílio obstétrico. Conforme os tutores, nasceram dois natimortos em estado de decomposição, não sendo observadas demais alterações clínicas dignas de nota na parturiente.

Num segundo momento, seis meses após o parto, a referida paciente foi encaminha para ovário-histerectomia eletiva. Por meio de exames clínico e laboratorial não foram observadas alterações e a paciente foi encaminhada para cirurgia. Após jejum sólido de oito horas e hídrico de duas horas, foi realizada medicação pré-anestésica com acepromazina $\left(0,05 \mathrm{mg} \cdot \mathrm{kg}^{-1} / \mathrm{IM}\right)$ e 
histopatolológicas

após 15 minutos, tricotomia abdominal ampla. Após o acesso venoso e início da fluidoterapia com ringer lactato de sódio (10 mL. $\left.\mathrm{kg}^{-1} \cdot \mathrm{h}^{-1} / \mathrm{IV}\right)$ a paciente foi induzida com propofol (4 mg.kg-1/IV), sendo intubada e mantida com isoflurano vaporizado em oxigênio a $100 \%$ em sistema aberto e respiração assistida. Associou-se infusão intravenosa contínua de citrato de fentanila (15 $\left.\quad \mu \mathrm{g} \cdot \mathrm{kg}^{-1} \cdot \mathrm{h}^{-1} / \mathrm{IV}\right) \quad \mathrm{e}$ administrou-se ampicilina sódica (20 mg. $\left.\mathrm{kg}^{-1} / \mathrm{IV}\right) 30$ minutos antes do início do procedimento cirúrgico.

Após antissepsia de pele, com a paciente em decúbito dorsal, foram colocados panos de campo e realizouse incisão longitudinal mediana retroumbilical de pele e subcutâneo com bisturi. Por punção acessou-se a cavidade abdominal e realizou-se a ampliação com tesoura de Mayo. O primeiro pedículo ovariano exposto foi 0 direito, sendo realizada a ruptura manual do ligamento suspensório, ligadura pelo método das três pinças modificado e a aplicação de uma ligadura circular e outra transfixante com poliglatina $910 \mathrm{n} \div 2-0$. Ao repetir-se a manobra no lado esquerdo verificou ausência do corno e tuba uterina correspondentes, porém a presença do rim e ovários ipsilaterais em posição anatômica. Realizou-se a ruptura manual do ligamento suspensório e mediante a técnica das três pinças modificada, realizou-se secção e ligadura conforme descrito anteriormente. Repetiu-se a manobra no corpo do útero e procedeu-se a sutura da parede abdominal e do espaço subcutâneo de maneira rotineira, assim como a dermorrafia. A paciente recebeu meloxicam $\left(0,1 \quad \mathrm{mg} \cdot \mathrm{kg}^{-1} / \mathrm{SID}\right)$ e a associação de dipirona sódica (25 mg.kg-1/QID) e N-butilescopolamina $(0,2$ $\left.\mathrm{mg} \cdot \mathrm{kg}^{-1} / \mathrm{QID}\right)$ durante dois dias, recuperando-se sem complicações, sendo as suturas de pele removidas no oitavo dia de pós-operatório. foram fixados em solução de formol $10 \%$ e encaminhados para exame histopatológico. O exame macroscópico revelou ausência completa do corno uterino e tuba uterina esquerdos, e a presença de ambos os ligamentos largos. Ao exame microscópico não foram encontradas alterações no corpo uterino, no corno uterino direito (Figura 1), tampouco nos ovários, incluindo o ipsilateal ao corno uterino ausente. No lugar deste, havia um feixe de tecido fibroso, rico em fibroblastos e colágeno, bem irrigado, localizado paralelamente a artéria uterina. $\mathrm{Em}$ maior aumento (Figura 2), entremeados aos feixes de tecido conjuntivo, havia grande quantidade de tecido adiposo, fibroblastos bem diferenciados e feixes de aglomerados de músculo liso de diferentes tamanhos.

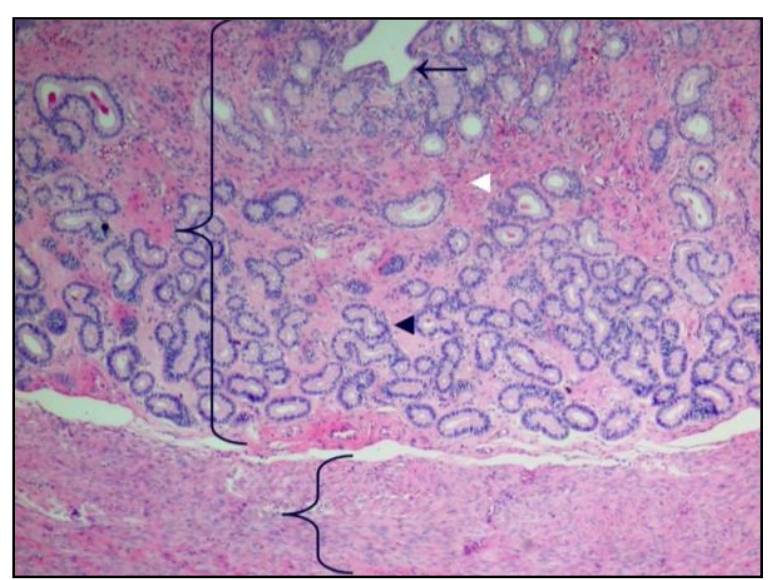

Figura 1 - Canino, fêmea, SRD, quatro anos com agenesia de corno uterino esquerdo. Histologia do corno uterino macroscopicamente presente (direito). Observar o endométrio (chave maior) constituído do epitélio de revestimento (seta), da lâmina própria rica em tecido fibrovascular maduro (cabeça de seta branca) e numerosas glândulas mucosas (cabeça de seta preta). Parte do miométrio está presente na porção inferior da figura (chave menor). Hematoxilina e eosina. 20X. 


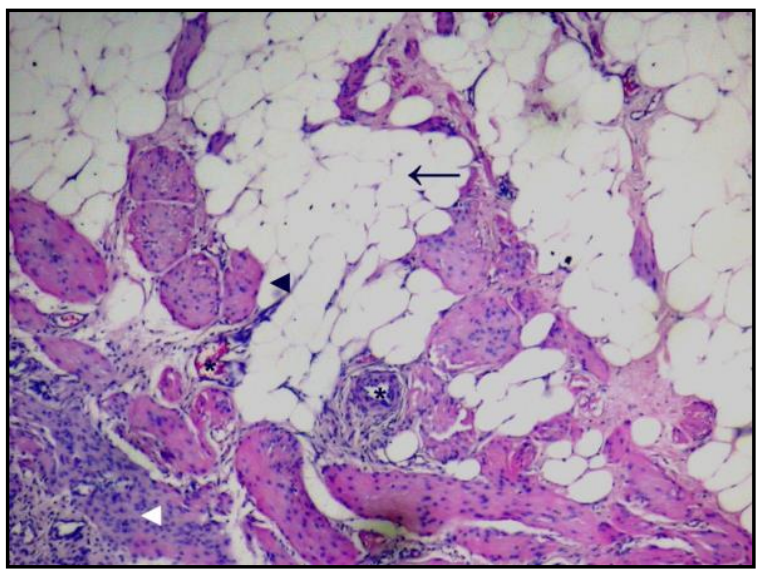

Figura 2 - Canino, fêmea, SRD, quatro anos com agenesia de corno uterino esquerdo. Histologia do tecido encontrado no local onde deveria estar - corno uterino macroscopicamente ausente (esquerdo). Em meio a grande quantidade de tecido adiposo (seta), há aglomerados esparsos de músculo liso (cabeça de seta preta), vasos sanguíneos (asteriscos) e feixes de tecido conjuntivo ricos em fibroblastos (cabeça de seta branca). Hematoxilina e eosina. 20X.

\section{DISCUSSÃO}

A agenesia de corno uterino consiste no não desenvolvimento ou no desenvolvimento incompleto dos ductos paramesonéfricos durante a formação fetal (Stone, 2003; Silva et al., 2010). A malformação pode ser parcial quando um segmento do corno uterino está ausente, ou completa no caso da inexistência total do mesmo (Nascimento e Santos, 2003), conforme observado no caso descrito. Embora Romagnoli e Schlafer (2006) salientem que em animais em que uma anormalidade é observada, outras alterações urogenitais, particularmente as ipsilaterais, podem estar presentes, isso não foi observado neste caso. McIntyre et al. (2010) relataram que metade dos afetados apresentaram agenesia renal ipsilateral. Além disso, também podem ser observadas ectopia ureteral contralateral, ausência de ligamento suspensório ipsilateral, cistos paraovarianos ipsilaterais e hérnias umbilicais, nenhum observado neste caso.

Em cães, os segmentos uterino e vaginal podem ter anormalidades de desenvolvimento que variam em severidade desde a hipoplaisa até a agenesia. Segundo McIntyre et al. (2010), o problema raramente constitui a queixa principal de infertilidade ou esterilidade, indo ao encontro deste caso, cujo tutor buscava apenas a ovário-histerectomia por conveniênca. Depois de realizado o diagnóstico da alteração, o responsável pelo animal reforçou que a paciente apresentava ciclos regulares e ausência de quaisquer alterações clínicas.

Anomalias uterinas podem ser considerados determinantes para distocia (Stone et al., 2003) ou infertilidade das afetadas (Stone et al., 2003; Güvenç et al., 2006), fato não observado neste caso, no qual o animal apresentou gestação e parto e não necessitou de assistência veterinária. 0 observado corrobora com Vince et al. (2011), que citam que embora o declínio da fertilidade seja possível, a ausência unilateral de corno uterino não impossibilita a gestação no lado oposto. Neste caso em particular, a ocorrência dos natimortos foi associada ao uso de progestágenos (González-Domínguez e Maldonado-Estrada, 2006), já que estes, dentre outros efeitos, podem provocar prolongamento da gestação e morte fetal.

Segundo Johnson et al. (2001), Nascimento e Santos (2003) e Colaço et al. (2012), as fêmeas acometidas podem apresentar ciclos estrais e exibir comportamento e cio normais, como verificado neste caso. Segundo England (2001), em caso de gestação, será observado pequeno número de filhotes, corroborando com o observado, quando nasceram apenas dois natimortos. Mclntyre et al. (2010) salientaram que além da gestação e concepção, estes 
histopatolológicas

animais podem ter lactação normal, como neste caso, e histórico de abortos, o que não foi verificado. Outra alteração ligada ao aspecto reprodutivo das afetadas é a mumificação de fetos ectópicos (Mclntyre et al., 2010; Cruz et al., 2016), não observada neste caso.

Anormalidade congênitas do útero em cães são raramente detectadas antes da puberdade. Além disso, um grande número de casos permanece desconhecido até 0 aparecimento de doenças secundárias, ou quando tentativas malsucedidas de reprodução da fêmea alertam o tutor a necessidade de exame reprodutivo detalhado do animal infértil (Colaço et al., 2012). Dentre os exames disponíveis estão a vaginoscopia, o exame ultrassonográfico e o radiográfico simples (England, 2001). Entretanto, a alteração constitui mais comumente um achado de necropsia (Colaço et al., 2012) ou durante a ovário-histerectomia (Wykes e Olson, 1996; Pinto Filho et al., 2001; Stone, 2003; Colaço et al., 2012; Aguirra et al., 2014), como no caso em questão. Ainda, o diagnóstico pode ser realizado durante a inspeção via laparotomia ou laparoscopia realizada devido a outras indicações (England, 2009).

O diagnóstico precoce da condição é muito importante, pois evita que os tutores mantenham um animal com doença congênita em reprodução, o que não ocorreu no caso em questão. Além disso, a identificação da alteração e a avaliação da importância desta permitem a recomendação adequada acerca da abordagem terapêutica, e estabelece o prognóstico para cada uma das condições específicas (Colaço et al., 2012).

Não é incomum o diagnóstico de anomalias uterinas durante procedimentos eletivos de ováriohisterectomia em cães (Romagnoli e Schlafer, 2006), como neste caso, quando deve ser realizada o referido procedimento, já que se sugere que a anomailia tem envolvimento hereditário e pode causar alterações na reprodução (Johnston et al., 2001). Entretanto, quando não há diagnóstico prévio, podem ocorrer complicações (Mclntyre et al., 2010), já que existe alteração da anatomia cirúrgica. Estas ocorrem especialmente quando aplicada a técnica do gancho, na qual inicialmente deve se identificar os cornos uterinos, e na sequência o corpo do útero e ovários (Stone, 2003). O fato não ocorreu no caso em questão, já que esta técnica não foi a aplicada.

Considerando que a alteração pode estar associada a outras, é importante que durante o procedimento o cirurgião realize a exploração cirúrgica e descarte outras anormalidades renais e ovarianas (Mclntyre et al., 2010), como realizado, sem observação de demais alterações. A exploração tornase especialmente importante no caso de agenesia renal ipsilateral, já que esta alteração requer técnicas de manejo específico no futuro (Mclntyre et al., 2010).

Embora tenham sido relatados que a ausência do ovário e tuba uterina ipsilaterais (Colaço et al., 2012) ou aumento de tamanho do ovário (Pagliuca et al., 2012), estas alterações não foram observadas. Neste caso, o rim ipsilateral estava presente, sem alterações, e foi utilizado como referencial para localização do ovário, conforme sugestão de Pinto Filho et al. (2001) e Vince et al. (2011), que facilitou a aplicação da hemostasia do complexo arterio-venoso ovariano pelo método das três pinças modificada. Ainda, segundo os últimos autores, em caso de agenesia renal, a bifurcação uterina poderia ter sido utilizada como referencial. Adicionalmente, é indicada a exploração abdominal completa a fim de descartar a presença de fetos ectópicos (Mclntyre et al., 2010). 
A alteração não comprometeu o tempo total de cirurgia, sendo o procedimento finalizado em 24 minutos. Associa-se o fato ao conhecimento prévio da alteração pelo cirurgião, a ausência de agenesia renal e a presença do ovário no sítio anatômico normal, que serviram de referência para confirmação da agenesia de corno uterino e realização do procedimento cirúrgico, conforme salientaram Pinto Filho et al. (2001). No período pósoperatório a paciente foi cuidadosamente monitorada e não foram identificadas alterações dignas de nota ou complicações, fato associado ao procedimento cirúrgico, que transcorreu sem demais complicações.

McIntyre et al. (2010) reportaram que histologicamente os componentes endometriais não foram identificados, sendo verificados tecido fibroso mesenquimal com alguma diferenciação muscular e vasos sanguíneos, assim como tecido similar ao ligamento largo. No lugar do corno uterino observa-se com fequência a ausência de tecido, ou ainda, constata-se a presença de estrutura filiforme. As informações corroboram com o observado no caso descrito, no qual se verificou um feixe de tecido fibroso, rico em fibroblastos e colágeno, bem irrigado, localizado paralelamente a artéria uterina em localização anatômica compatível com a do corno uterino ausente. Ainda, entremeados aos feixes de tecido conjuntivo, observou-se grande quantidade de tecido adiposo, fibroblastos bem diferenciados e feixes de aglomerados de músculo liso de diferentes tamanhos, conforme destacado pelos autores.

A causa e a herdabilidade das anomalias uterinas em cães e gatos não é conhecida. Permanece incerto se as influências genéticas, endócrinas ou ambientais estão envolvidas, ou se as anormalidades são condições primárias, que se desenvolvem secundariamente à ausência do mesonefron, ou ainda, que sejam comprometidas pela ausência de suprimento sanguíneo (Johnston et al., 2001). Estudos em ratos sugeriram que a herança de anormalidades dos ductos Müllerianos pode ser poligênica ou multifatorial (Simpson, 1999). Como muitas variáveis podem estar envovidas no desenvolvimento da alteração, neste caso a causa não pode ser identificada.

\section{CONCLUSÃO}

Cadelas com agenesia de corno uterino podem apresentar ciclos reprodutivos regulares, gestação e parto eutócio, apesar do reduzido número de filhotes. O conhecimento da anatomia cirúrgica foi eficaz na realização do diagnóstico da alteração e execução da ovário-histerectomia.

\section{REFERÊNCIAS}

AGUIRRA, L.R.V.M; PEREIRA, W.L.A.; MONGER, S.G.B. et al. Aplasia de unicorno uterino em cadela - Relato de caso. Revista Brasileira de Medicina Veterinária, Rio de Janeiro, v.36, n.4, p.351-354, 2014.

COLAÇO, B.; PIRES, M.A.; PAYANCARREIRA, R. Congenital aplasia of the uterine-vaginal segment in dogs. In: PEREZ-MARIN, C.C. A Bird-eye View of Veterinary Medicine. Rijeka: Intech, 2012. Cap.10, p.165-178.

ENGLAND, G.C.W. Infertility in the bitch and queen. In: NOAKES. D.E., PARKINSON, T.J, ENGLAND. In: Arthur's Veterinary Reproduction and Obstetrics. 8.ed. China: Elsevier, 2001. Cap.28, p.639-670.

ENGLAND, G.C.W. 2009. Infertility and subfertility in the bitch and queen. In: NOAKES D.E., PARKINSON T.J. \& ENGLAND G.C.W. (Eds). Veterinary reproduction and obstetrics. 9th ed. London: Saunders Elsevier, pp.647-648. GONZÁLEZ-DOMÍNGUEZ, M.S.; MALDONADO-ESTRADA, J.G. Gestación prolongada asociada con la prescripción inadecuada de 
medroxiprogesterona acetato. ¿Es racional y ético el uso de progestágenos exógenos en perras? Revista Coombiana de Ciencias Pecuarias, Medellín, v.19, n.4, p. 442-450, 2006. JOHNSTON, S.D., KUSTRITZ, M.V.R., OLSON, P.N.S. Canine and Feline Theriogenology. 2.ed. Philadelphia: WB Saunders Co, 2001. 592p.

LYLE, S.K. Disorders of sexual development. In: KUSTRITZ, M.V.R. Small Animal Theriogenology. 2.ed. St. Louis, MO: Butterworth-Heinemann; 2003. Cap.1, p.1-32.

MCINTYRE, R.L.; LEVY, J.K.; ROBERTS, J.F. et al. Developmental uterine anomalies in cats and dogs undergoing elective ovariohysterectomy. Journal of American Veterinary Medical Association, Schaumburg, v. 237, n.5, p.542-546, 2010.

NASCIMENTO, E.F.N.; SANTOS, R.L. Patologia do Sistema Genital Feminino. In: _. Patologia da Reprodução dos Animais Domésticos. 2.ed. Rio de Janeiro: Guanabara Koogan, 2003. Cap.5, p.15-89.

PAGLIUCA, T.C.L.; MARQUES, J.M.V.; FILADELPHO, A.L. Malformações congênitas na ovariohisterectomia em cadelas: útero unicórnio - Relato de caso. Revista Científica Eletrônica de Medicina Veterinária, Garça, Ano X, n.19, 2012.

PINTO FILHO, S.T.L.; CUNHA, O.; RAISER, A.G. et al. Agenesia unilateral de corno uterino em cadela - Relato de caso. Arquivo de Ciências Veterinárias e Zoologia da UNIPAR, Umuarama, v.4, p.77-79, 2001.

ROMAGNOLI, S., SCHLAFER, D.H. Disorders of sexual differentiation in puppies and kittens: a diagnostic and clinical approach. Veterinary Clinics of North America Small Animal Practice. v.36, p.573-606, 2006.

STONE, E.A. Ovary and uterus. In: SLATTER, D.H. Textbook of small animal surgery, 3.ed. Philadelphia: Saunders, 2003, Cap.98, p.1487-1502.
SILVA, D.M., BATISTA, F.G., OLIVEIRA, D.R., et al. Agenesia unilateral de rim, corno uterino e ovário em cadela - Relato de caso. Jornal Brasileiro de Ciência Animal. v.3, n.16, p. 227-230, 2010.

SIMPSON, J.L. Genetics of the female reproductive ducts. American Journal of Medicine Genetic. v.89, p.224-239, 1999.

VINCE, S.; ZEVRNJA, B.; BECK, A. et al. Unilateral segmental aplasia of the uterine horn in a gravid bitch - Case report. Veterinarski Arhiv, Croatia. v.81. n.5, p.691-698, 2011.

WYKES, P.M.; OLSON, P.N. Moléstias do útero. In: BOJRAB, M.J. Mecanismos e moléstias na cirurgia dos animais domésticos. 2. ed. São Paulo: Manole, 1996, Cap.83, p.665669. 